\title{
Prognostic role of DFNA5 in head and neck squamous cell carcinoma revealed by systematic expression analysis
}

Zhiguo Liu ${ }^{1,2+}$, Hongyan Liu ${ }^{2,3+}$, Qian Dong ${ }^{1,2}$, Hongyu Li ${ }^{1,2}$, Bin Zhang ${ }^{1,2}$, Yufeng Liü ${ }^{4}$, Limei Zhong ${ }^{5^{*}}$ and Haikuo Tang ${ }^{1,2^{*}}$

\begin{abstract}
Background: The gasdermin E gene (GSDME, also known as DFNA5) is mutated in familial aging-related hearing loss. Recent studies have also revealed that the expression of DFNA5 is suppressed in many cancer types; however, little is known about the function of DFNA5 in head and neck squamous cell carcinoma (HNSCC). Accordingly, the aim of the present study was to evaluate the expression of DFNA5 and explore its prognostic value in HNSCC.

Result: We used a set of bioinformatics tools, including Oncomine, TIMER, TISIDB, cBioPortal, and GEPIA, to analyze the expression of DFNA5 in patients with HNSCC from public databases. Kaplan-Meier plotter was used to evaluate the potential prognostic significance of DFNA5. DFNA5 mRNA levels were significantly higher in HNSCC tissues than in normal tissues, and high DFNA5 expression was correlated with worse survival. Gene Ontology and Kyoto Encyclopedia of Genes and Genomes pathway analyses showed that DFNA5 expression has a strong positive correlation with cell adhesion and the integrin signaling pathway, whereas its expression was negatively correlated with the levels of infiltrating $B$ cells (cor $=-0.223, P=8.57 \mathrm{e}-07$ ) and CD8 T cells (cor $=-0.223, P=2.99 \mathrm{e}-07$ ).
\end{abstract}

Conclusion: This study demonstrates that DFNA5 expression has prognostic value for HNSCC patients. Moreover, these results suggest that regulation of lymphocyte infiltration is the mechanism underlying the function of DFNA5 in HNSCC.

Keywords: DFNA5, Head and neck cancer, Survival prognosis, Lymphocyte infiltration

\section{Introduction}

Head and neck cancers include a wide variety of cancers varying in location and histological types. One of the subcategories is head and neck squamous cell carcinomas (HNSCCs), which include tumors of the nasal cavity, nasopharynx, oral cavity, oropharynx, hypopharynx,

\footnotetext{
* Correspondence: 854458483@qq.com; tanghk@mail.sysu.edu.cn ${ }^{+}$Zhiguo Liu and Hongyan Liu contributed equally to this work. ${ }^{5}$ Department of Laboratory Medicine, Guangdong Second Provincial General Hospital, No. 466 Xingang Middle Road, Haizhu District, Guangzhou 510317, China

'Department of Oral and Maxillofacial Surgery, Hospital of Stomatology, Sun Yat-sen University, 56 Lingyuanxi Road, Guangzhou 510055, China Full list of author information is available at the end of the article
}

and larynx [1,2]. Approximately 600,000 new cases of HNSCC are diagnosed each year worldwide, often at an advanced stage. Moreover, epidemiological data indicate that the incidence of oral cancer is increasing year after year [3]. Although the use of combination therapy (i.e., surgical techniques, chemotherapy, and radiation therapy) has significantly improved local control and the overall quality of life of HNSCC patients, their survival rate has only increased slightly in the past 20 years [4]. Therefore, it is imperative to understand the potential molecular carcinogenic pathways of HNSCC, which is expected to identify markers that can help to improve the diagnosis, treatment, and prevention of the disease.

(C) The Author(s). 2021 Open Access This article is licensed under a Creative Commons Attribution 4.0 International License, which permits use, sharing, adaptation, distribution and reproduction in any medium or format, as long as you give appropriate credit to the original author(s) and the source, provide a link to the Creative Commons licence, and indicate if changes were made. The images or other third party material in this article are included in the article's Creative Commons licence, unless indicated otherwise in a credit line to the material. If material is not included in the article's Creative Commons licence and your intended use is not permitted by statutory regulation or exceeds the permitted use, you will need to obtain permission directly from the copyright holder. To view a copy of this licence, visit http://creativecommons.org/licenses/by/4.0/ The Creative Commons Public Domain Dedication waiver (http://creativecommons.org/publicdomain/zero/1.0/) applies to the data made available in this article, unless otherwise stated in a credit line to the data. 
Gasdermin E (GSDME), also known as deafness autosomal dominant 5 (DFNA5), was identified as a gene involved in an autosomal dominant form of inherited hearing impairment in 1998 [5]. Interestingly, subsequent reports showed that DFNA5 also plays a role in tumor biology. Specifically, DFNA5 has been suggested to act as a tumor suppressor, since it was shown to inhibit the colony formation and cell proliferation of gastric cancer, melanoma, and colorectal cancer cells, and could also suppress the aggressive behavior of breast cancer [6]. Recently, some chemotherapeutic agents such as cisplatin [7], L61H10 [8], and lobaplatin [9] were shown to be effective against esophageal cancer, lung cancer, and colon cancer, respectively, by inducing a DFNA5-dependent pyroptosis effect. These results have led to a new understanding of cancer chemotherapy, while indicating that DFNA5 is a potential target for cancer treatment. However, the role of DFNA5 in HNSC $\mathrm{C}$ development and progression remains unknown.

In this work, we applied a wide range of comprehensive bioinformatics tools to assess the expression levels and potential function of DFNA5, as well as determine its prognostic value in human HNSCC.

\section{Materials and methods Oncomine}

In order to evaluate the expression of DFNA5 in different tumors, Oncomine (https://www.oncomine.org/ resource/login.html) is a publicly accessible online cancer microarray database that can be used for research related to genome-wide expression analysis. We used this database to extract data on the DFNA5 mRNA levels (log2-transformed) in HNSCC and neighboring normal tissues. The criteria for identifying a significant difference were a $P$-value $<1 \mathrm{E}-4$, fold change $>2$, and the gene ranks in the top $10 \%[10]$.

\section{Timer}

TIMER is a web server for the comprehensive analysis of expression pattern and tumor-infiltrating immune cells. It offers six tumor-infiltrating immune subsets precalculated for 10,897 tumors from 32 cancer types (https:// cistrome.shinyapps.io/timer/) [11]. TIMER web server allows users to input function-specific parameters, with resulting figures dynamically displayed to conveniently access the tumor immunological, clinical, and genomic features.

\section{TISIDB}

TISIDB is an integrated repository portal for tumorimmune system interactions, which can also be used for the systematic testing of molecular features of such interactions TISIDB is the most comprehensive database for tumor and immune system interactions. In TISIDB, users can cross check the gene of interest through knowledge from different platforms and obtain high quality visualization results for their publications (http://cis.hku. hk/TISIDB/) [12]. In present study, we use TISIDB to predict the prognosis evaluation.

\section{cBioPortal}

cBioPortal includes tools for the visualization, analysis, and downloading of large-scale cancer genomics datasets. In order to regulatory network of DFNA5, we used cBioPortal to identify genes positively related to DFNA5 expression in HNSCC [13].

\section{GEPIA}

GEPIA (http://gepia.pku.cn/) is a newly developed interactive web server for analyzing the RNA sequencing expression data of 9736 tumors and 8587 normal samples from the TCGA and the GTEx projects, using a standard processing pipeline. GEPIA provides customizable functions such as tumor/normal differential expression analysis, profiling according to cancer types or pathological stages, patient survival analysis, similar gene detection, correlation analysis and dimensionality reduction analysis. In present study, GEPIA was used to analysis differential expression and survival analyses [14].

\section{Kaplan-Meier plotter}

The Kaplan Meier plotter is capable to assess the effect of $54 \mathrm{k}$ genes (mRNA, miRNA, protein) on survival in 21 cancer types. Sources for the databases include GEO, EGA, and TCGA. Primary purpose of the tool is a metaanalysis based discovery and validation of survival biomarkers. Kaplan-Meier plotter (http://kmplot.com/ analysis/) was used to evaluate the potential prognostic significance of DFNA5 [15]. The hazard ratio (HR) with a 95\% confidence interval and the $P$-value (log-rank) were calculated. To research the relationship between the DFNA5 expression level and specific clinical characteristics of HNSCC patients. Enter DFNA5 in the website of Kaplan-Meier plotter, select HNSCC in Pancancer RNA-seq database, and then select the corresponding index (as shown in Table 2) in the right-hand border, click draw Kaplan-Meier plot to record the corresponding results.

\section{Cytoscape}

Cytoscape (version 3.4.0) is an open-source bioinformatics software tool for visualizing molecular interaction networks. Its plug-in, MCODE, is used for ranking nodes in a network according to their network features [16]. Presently, we use Cytoscape to study the regulatory network of DFNA5 in HNSCC. 


\section{David}

DAVID (http://david.abcc.ncifcrf.gov/) is a tool that is widely used to reveal the biological significance of gene groups. After selecting co-expressed genes, Gene Ontology (GO) analysis was performed to assess their associated biological processes (BP), cellular components (CC), and molecular functions (MF) [17]. The Kyoto Encyclopedia of Genes and Genomes (KEGG) pathway database was used to identify biological pathways enriched with the co-expressed genes [18].

\section{UALCAN}

UALCAN (http://ualcan.path.uab.edu) is an interactive web resource that can combine clinical data for 31 cancer types with corresponding RNA-seq data from the TCGA database. It is built on PERL-CGI with high quality graphics using javascript and CSS. As such, it can be used to analyze relative gene transcript levels between tumors and normal samples, and the relationship between these levels and clinicopathological parameters [19].

\section{Realtime PCR}

We use HNSCC cell lines for in vitro gene expression validation, such as SCC-15 (ATCC CRL-1623), Cellosaurus HN6 (CVCL_8129), and Human oral squamous cell carcinoma (HSC-3); Human normal oral keratinocytes (NOK) as control cells. Total RNA was isolated from cell lines using TRIzol (Takara). RNA was then converted to cDNA using PrimeScript RT Master Mix (Takara). qPCR was performed on a Bio-Rad Thermal Cycler using SYBR Premix Ex Taq II (Takara) with primers against human DFNA5. For-ACATGCAGGT CGAGGAGAAGT; Rev-TCAATGACACCGTAGGCA ATG. Relative expression was calculated using the deltadelta $\mathrm{Ct}$ method and normalized to the reference gene GADPH For-ACAACTTTGGTATCGTGGAAGG; RevGCCATCACGCCACAGTTTC.

\section{Statistical analysis}

The survival curves were generated via Kaplan- Meier plots and PrognoScan database are displayed with HR and $\mathrm{P}$ or Cox $P$-values from a log-rank test. Gene expression data in the Oncomine database was analyzed using $p$-value, fold change, and mRNA data type. Spearman correlation analysis was used to evaluate the correlation of gene expression in TIMER, LinkedOmics and UALCAN databases. ${ }^{*} P<0.05, * P<0.01, * * * P<$ 0.001 was considered statistically significant.

The list of experiments were listed in Table 1.

\section{Results}

DFNA5 expression is upregulated in human HNSCC

The analysis of microarray data from HNSCC and adjacent normal tissues in the Oncomine database showed that DFNA5 expression was upregulated in HNSCC (Fig. 1A). This pattern was confirmed by the analysis of gene expression data deposited in TIMER (Fig. 1B). In addition to bulk analysis of HNSCC data, we also used Oncomine to compare DFNA5 expression in healthy and cancer tissues from datasets corresponding to different HNSCC subtypes. These results showed that DFNA5 upregulation is a general characteristic of HNSCC (Fig. $1 \mathrm{C}-\mathrm{F})$. Further subgroup analysis of various clinicopathological features of HNSCC patients from the TCGA database revealed that in subgroup analyses based on gender, age, race, disease stage, tumor grade, human papillomavirus infection, and nodal metastasis status, the transcription level of DFNA5 in HNSCC patients was consistently significantly higher than that in healthy subjects (Sup Fig. 1). Furthermore, we used HNSCC cell lines for in vitro validation, demonstrating a significant increase in the level of DFNA5 compared to that of control cells (Fig. 1G). Collectively, these results suggest that the expression of DFNA5 may be a potential diagnostic marker of HNSCC.

\section{High DFNA5 expression predicts poor prognosis in HNSCC patients}

We speculated that the expression of DFNA5 is related to the prognosis of HNSCC patients. To test this hypothesis, we evaluated the prognostic value of DFNA5 using the Kaplan-Meier plotter, TISIDB, and UALCAN (Fig. 2A-C, respectively). All three analyses showed that high DFNA5 expression levels are associated with a poor prognosis in HNSCC patients. Moreover, this association was consistent regardless of tumor grade (Fig. 2D), race (Fig. 2E), or gender (Fig. 2F). To further understand this correlation and assess the potential underlying mechanism, we utilized the Kaplan-Meier plotter to explore the relationship between the DFNA5 expression level and specific clinical characteristics of HNSCC patients. As

Table 1 The list of experiments

\begin{tabular}{ll}
\hline Methods (database) & Results \\
\hline Oncomine, TIMER, GEPIA, UALCAN, qRT-PCR & Expression of DFNA5 \\
Kaplan-Meier plotter, TISIDB, and UALCAN & Prognosis value of DFNA5 \\
CBioPortal, UALCAN, DAVID, CytoHubba & Regulatory network of DFNA5 \\
TIMER & Infiltration of immune cells \\
\hline
\end{tabular}




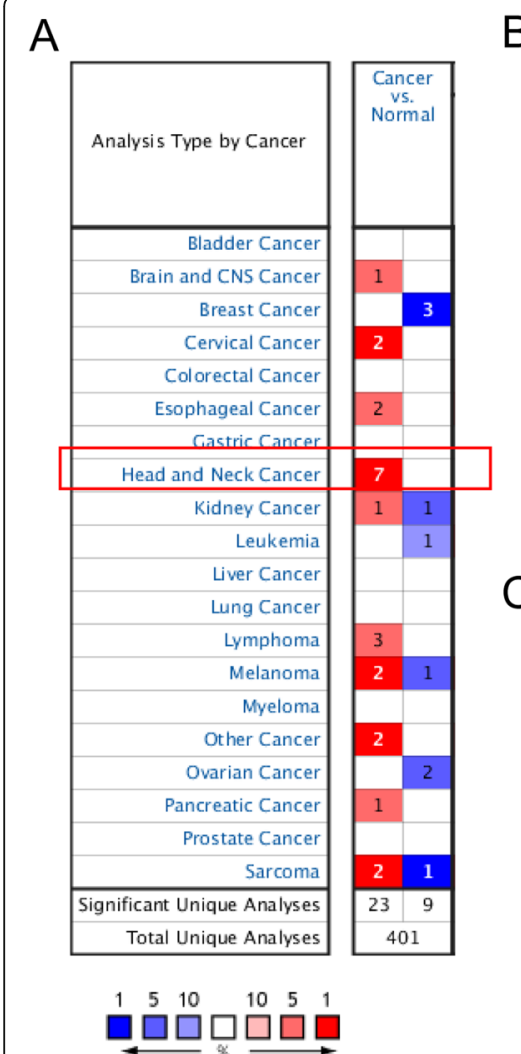

E

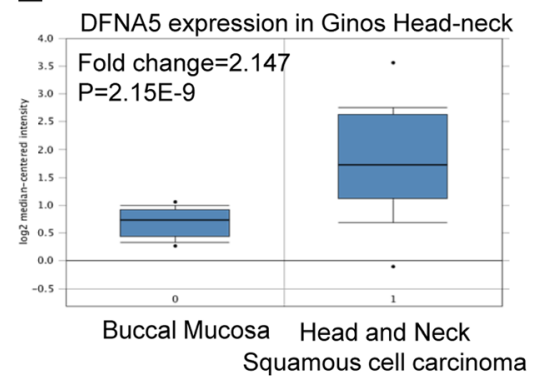

B

C
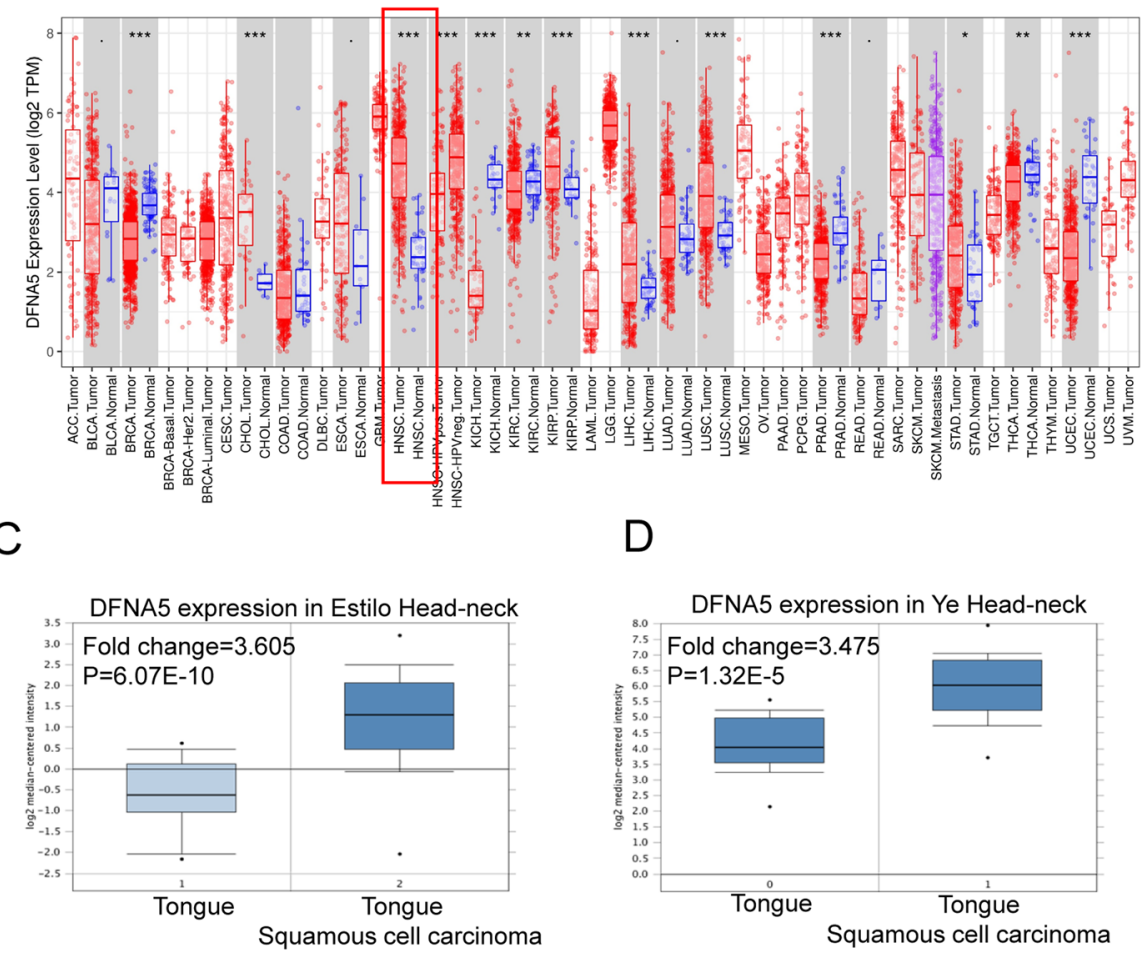

$\mathrm{F}$

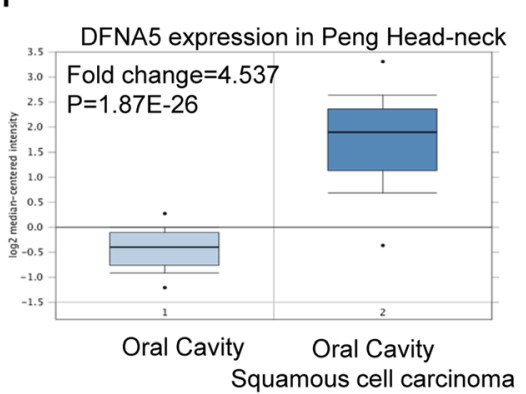

G

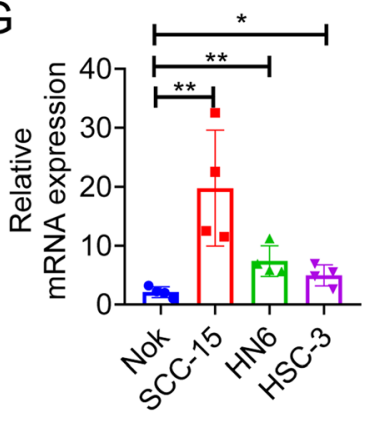

DFNA5

Fig. 1 DFNA5 expression in human head and neck squamous cell carcinoma. (A-B) The expression level of DFNA5 in HNSCCs and healthy tissues as calculated from the gene expression data of the Oncomine database (A), and TIMER database (B). (C-F) Analysis of DFNA5 expression in different subtypes of HNSCC using gene expression data from the Oncomine database. (G) In vitro validation of the expression level of DFNA5 in the HNSCC cell lines SCC-15 (ATCC CRL-1623), Cellosaurus HN6 (CVCL_8129), and human oral squamous cell carcinoma (HSC-3); human normal oral keratinocytes (NOKs) were used as control cells. ${ }^{*} \mathrm{P}<0.05,{ }^{* *} \mathrm{P}<0.01,{ }^{* * *} \mathrm{P}<0.001$

shown in Table 2, high expression of DFNA5 was significantly correlated with worse overall survival (OS) only in male patients $(P=0.041)$. Moreover, racial background did not have a statistically significant effect. With respect to tumor stage, DFNA5 overexpression was associated with poorer OS in HNSCC patients at all stages except for stage 3. More importantly, we found that high expression of DFNA5 corresponded with a worse OS in patients whose tumors were infiltrated by immune cells such as basophils, B-cells, T-cells, and macrophages (Table 2). These results suggest that DFNA5 may be a reliable biomarker for HNSCC prognosis, at least in male patients.

\section{DFNA5 regulates cell adhesion in HNSCC}

Lists of the top 200 genes co-expressed with DFNA5 were created by analyzing TGCA datasets with cBioPortal (Fig. 3A) and UALCAN (Fig. 3B). Comparison of the two lists revealed 125 common co-expressed genes (Fig. 3C). DAVID was then used to perform functional annotation of these 125 genes. GO analysis indicated that these genes were mainly involved in biological processes 

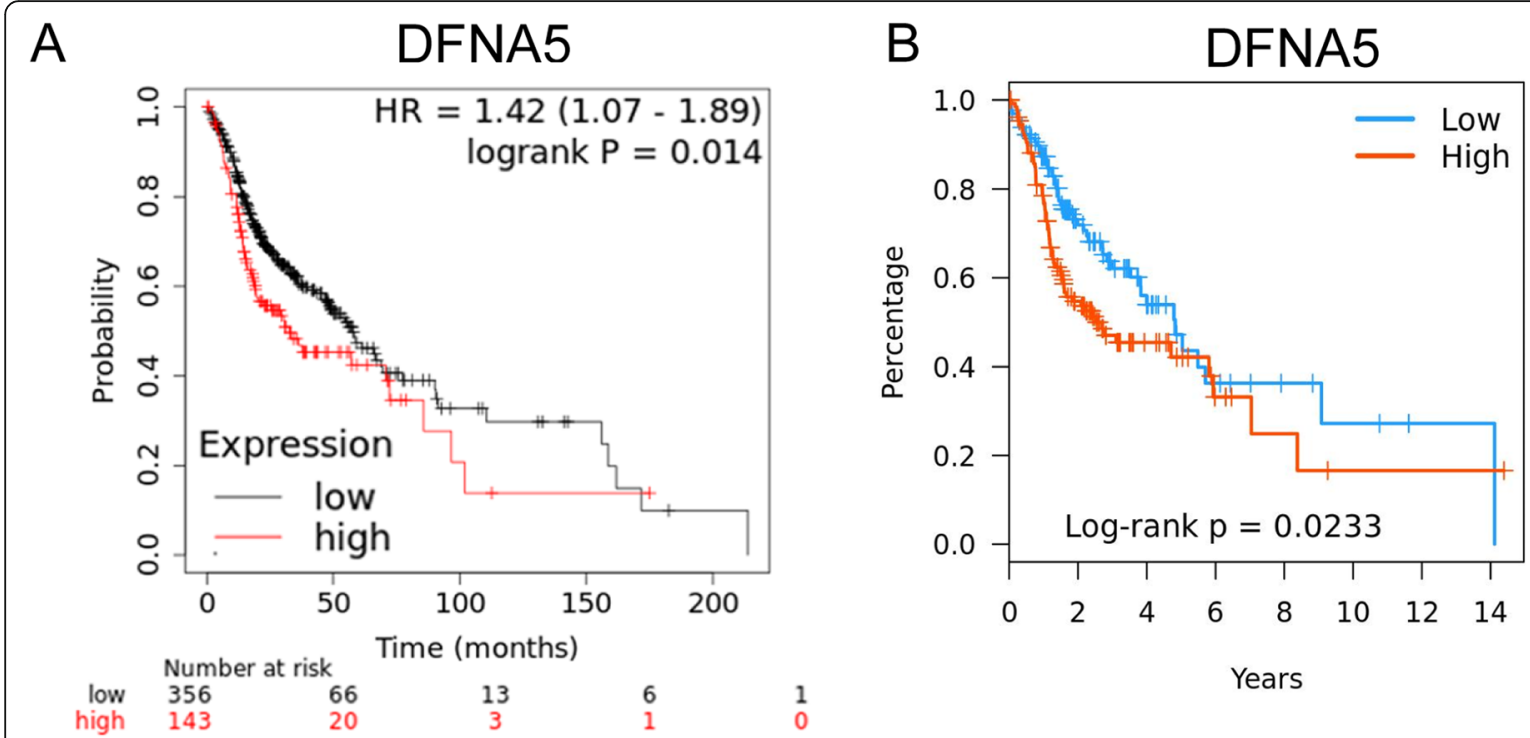

C

Effect of DFNA5 expression level on HNSC patient survival

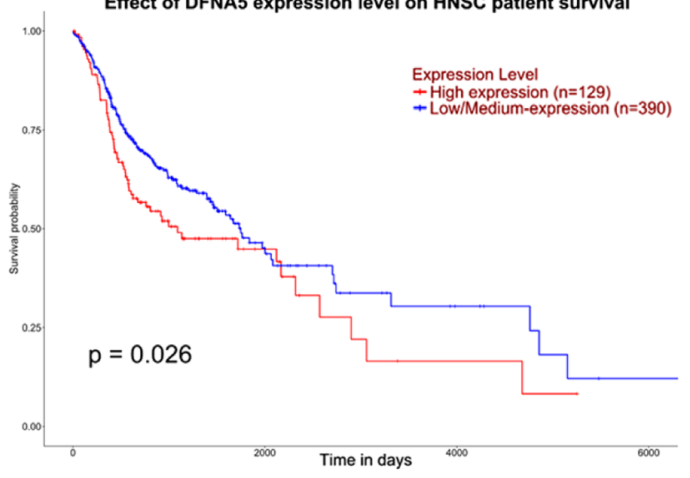

$\mathrm{D}$

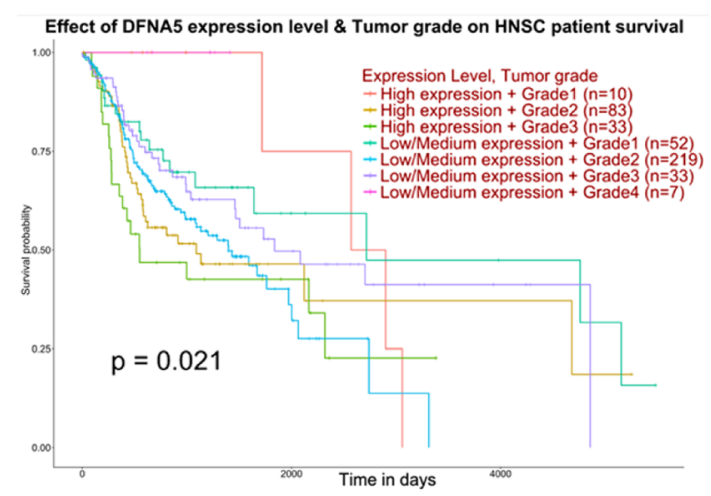

$E$

Effect of DFNA5 expression level \& Race on HNSC patient survival

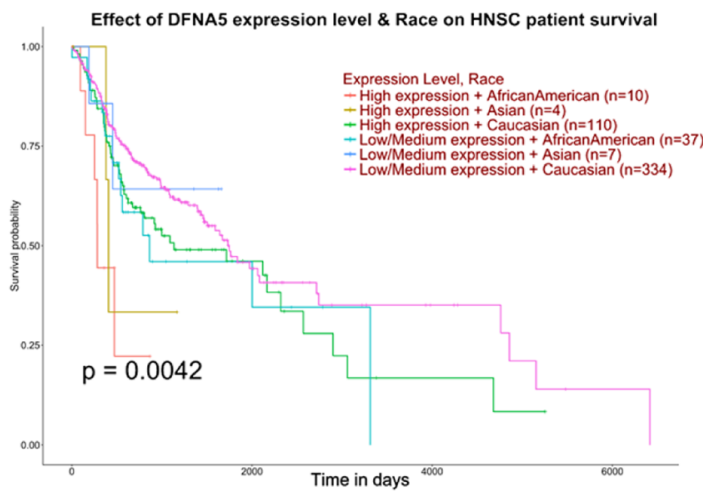

$\mathrm{F}$

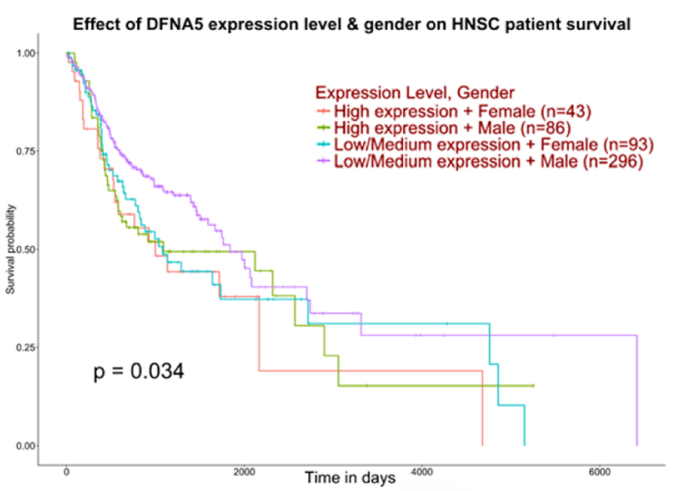

Fig. 2 DFNA5 prognostic value in human head and neck squamous cell carcinoma. (A-C) Correlation between overall survival (OS) and DFNA5 expression in patients with HNSCC as assessed using the Kaplan-Meier plotter (A), TISIDB (B), and UALCAN (C). (D-F) Correlation between OS and DFNA5 expression for patients of different tumor grades (D), races (E), and genders (F) as assessed using UALCAN. ${ }^{*} P<0.05$ 
Table 2 Correlation between DFNA5 expression and clinical prognosis in head and neck squamous cell carcinoma with different clinicopathological factors using the Kaplan-Meier plotter

\begin{tabular}{|c|c|c|c|c|}
\hline \multicolumn{2}{|c|}{ Clinicopathological characteristics } & \multicolumn{3}{|c|}{ OS survival } \\
\hline & & \multirow{2}{*}{$\begin{array}{l}\mathbf{N} \\
133\end{array}$} & \multirow{2}{*}{$\begin{array}{l}\text { Hazard ratio } \\
1.56(0.95-2.55)\end{array}$} & \multirow{2}{*}{$\frac{P \text {-value }}{0.076}$} \\
\hline Gender: & Female & & & \\
\hline & Male & 366 & $1.43(1.01-2.03)$ & 0.041 \\
\hline \multirow[t]{3}{*}{ Race: } & White & 426 & $0.35(1.01-1.82)$ & 0.044 \\
\hline & black/African American & 47 & $3.4(1.3-8.91)$ & 0.0084 \\
\hline & 1 & 25 & $7.29(0.76-70.09)$ & 0.044 \\
\hline \multirow[t]{4}{*}{ Stage } & 2 & 69 & $0.45(0.2-1)$ & 0.043 \\
\hline & 3 & 78 & $1.55(0.68-3.55)$ & 0.29 \\
\hline & 4 & 259 & $1.75(1.21-2.53)$ & 0.0027 \\
\hline & 1 & 61 & $1.94(0.8-4.7)$ & 0.14 \\
\hline \multirow[t]{4}{*}{ Grade: } & 2 & 298 & $1.29(0.91-1.82)$ & 0.15 \\
\hline & 3 & 119 & $2.25(1.29-3.92)$ & 0.0033 \\
\hline & 4 & 7 & & \\
\hline & high & 251 & $1.34(0.91-1.98)$ & 0.14 \\
\hline \multirow[t]{2}{*}{ Mutation burden: } & low & 243 & $1.95(1.29-2.97)$ & 0.0014 \\
\hline & enriched & 243 & $1.7(1.11-2.6)$ & 0.013 \\
\hline \multirow[t]{2}{*}{ Basophils: } & decreased & 254 & $1.22(0.83-1.79)$ & 0.3 \\
\hline & enriched & 238 & $2.07(1.31-3.28)$ & 0.0015 \\
\hline \multirow[t]{2}{*}{ B-cells: } & decreased & 259 & $1.16(0.77-1.73)$ & 0.48 \\
\hline & enriched & 316 & $1.66(1.16-2.37)$ & 0.0048 \\
\hline \multirow[t]{2}{*}{ CD4+ memory T-cells: } & decreased & 181 & $1.3(0.84-2.01)$ & 0.24 \\
\hline & enriched & 305 & $1.55(1.05-2.27)$ & 0.025 \\
\hline \multirow[t]{2}{*}{ CD8+ T-cells: } & decreased & 192 & $1.62(1.04-2.51)$ & 0.03 \\
\hline & enriched & 215 & $1.55(1.05-2.27)$ & 0.025 \\
\hline \multirow[t]{2}{*}{ Eosinophils: } & decreased & 282 & $1.53(1.07-2.19)$ & 0.02 \\
\hline & enriched & 205 & $1.49(0.91-2.38)$ & 0.12 \\
\hline \multirow[t]{2}{*}{ Macrophages: } & decreased & 292 & $1.34(0.93-1.94)$ & 0.12 \\
\hline & enriched & 313 & $1.62(1.15-2.27)$ & 0.0053 \\
\hline \multirow[t]{2}{*}{ Mesenchymal stem cells: } & decreased & 184 & $1.33(0.85-2.07)$ & 0.21 \\
\hline & enriched & 129 & $1.54(0.94-2.52)$ & 0.082 \\
\hline \multirow[t]{2}{*}{ Natural killer T-cells: } & decreased & 368 & $1.36(0.97-1.9)$ & 0.077 \\
\hline & enriched & 322 & $1.43(1.01-2.01)$ & 0.04 \\
\hline \multirow[t]{2}{*}{ Regulatory T-cells: } & decreased & 175 & $1.54(0.95-2.48)$ & 0.074 \\
\hline & enriched & 381 & $1.53(1.11-2.11)$ & 0.0082 \\
\hline \multirow[t]{2}{*}{ Type 1 T-helper cells: } & decreased & 116 & $1.38(0.8-2.39)$ & 0.24 \\
\hline & enriched & 473 & $1.39(1.04-1.85)$ & 0.024 \\
\hline Type 2T-helper cells: & decreased & 24 & $4.84(0.8-29.1)$ & 0.057 \\
\hline
\end{tabular}

of locomotion, cell adhesion, and cell migration (Fig. 3D), which was consistent with the enrichment in the respective cellular components and the proposed molecular functions (Fig. 3E). In addition, KEGG pathway analysis showed enrichment in pathways of focal adhesion and the interaction between the actin cytoskeleton and the extracellular matrix receptor (Fig. 3F). Meanwhile, GSEA analysis also showed that the high expression group of DFNA5 was related to focal adhesion pathway (Fig. 3G). Collectively, these data suggest an essential role of DFNA5 in regulating cell adhesion in HNSCC. 


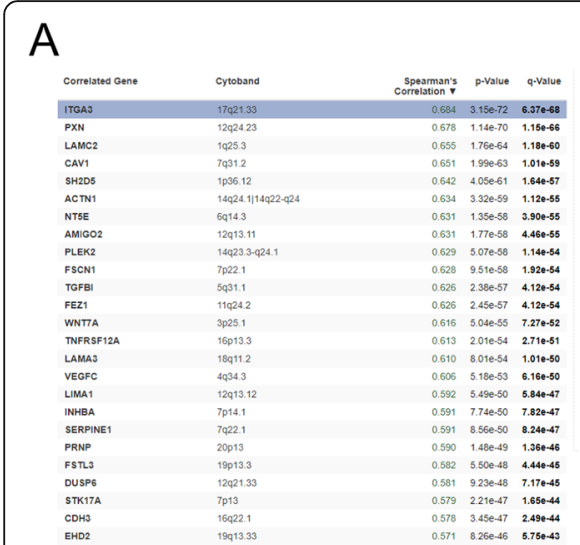

B

C
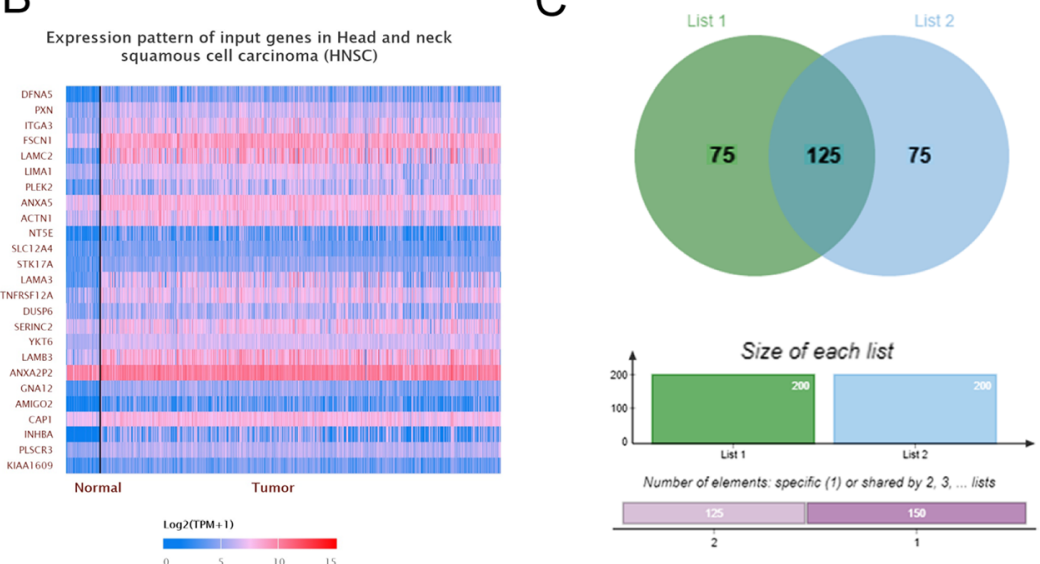

Number of elements: specific (1) or shared by 2,3,... lists

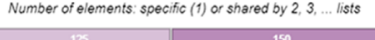

$\mathrm{E}$
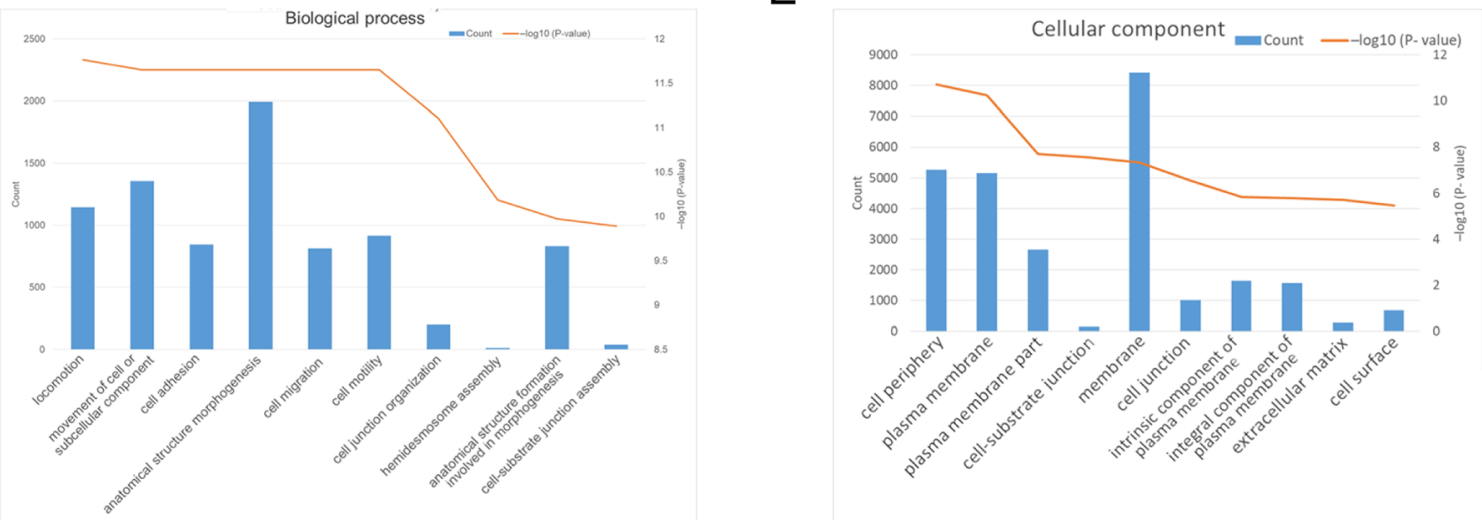

$\mathrm{F}$

G
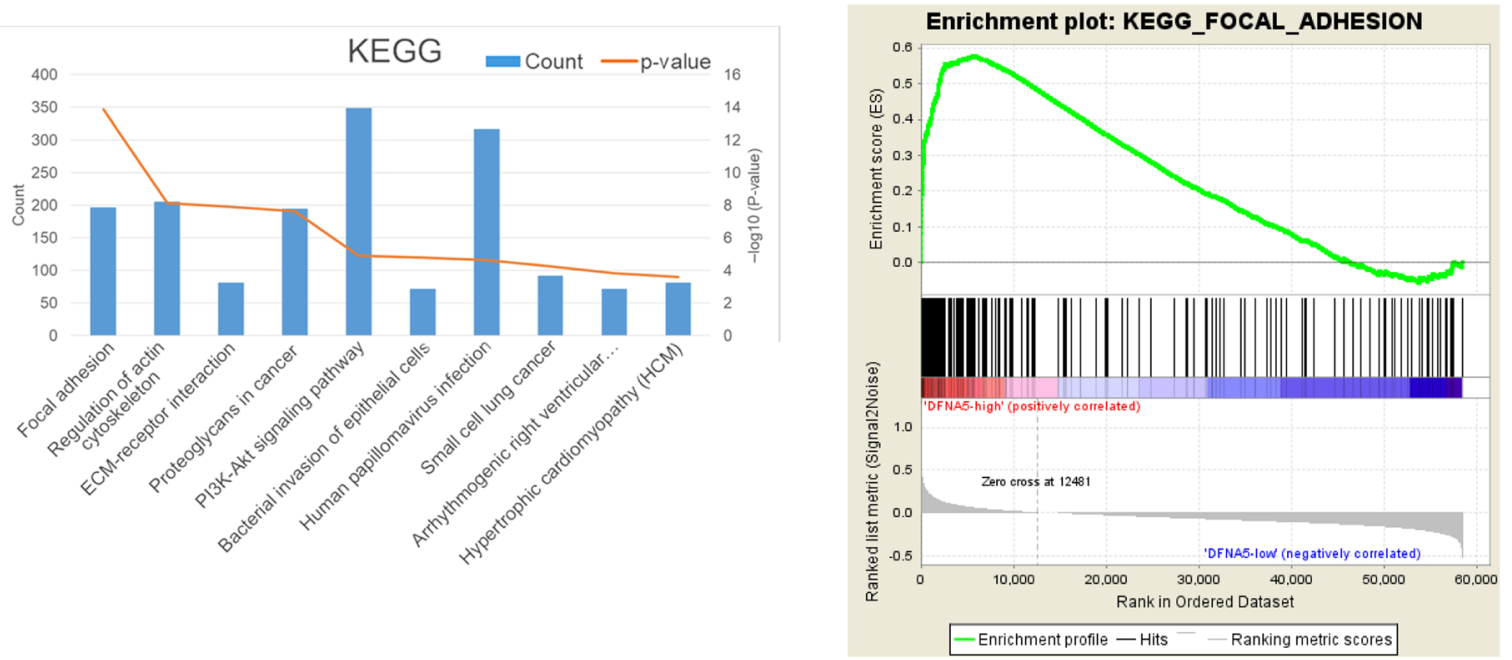

Fig. 3 Enrichment function analyses of co-expressed genes indicating an association of DFNA5 with cell adhesion. (A, B) Lists of the top 200 genes positively correlated with DFNA5 expression as determined using cBioPortal (A) and UALCAN (B). (C) Venn diagram of the two lists showing an intersection containing 125 genes. (D-F) GO enrichment in biological processes (D), and cellular components (E), for the 125 common genes. (F) KEGG pathway enrichment analysis of the 125 common genes. (G) GSEA analysis showed that the high expression group of DFNA5 was correlated with focal adhesion pathway 
Identification and analysis of DFNA5-related hub genes CytoHubba, a Cytoscape plug-in, was used to identify potential hub genes for DFNA5 function based on the density of maximum neighborhood component. The top 10 genes in the network included ITGB1, ITGA3, ITGB4, ITGA6, PXN, ITGA5, LAMC2, LAMA3, PLEC, and
$L A M B 3$ (Fig. 4A), which were belong to these positively co-expressed genes. GEPIA was then used to analyze the expression level of each hub gene and its correlation with OS. All genes except for PLEC had higher expression levels in HNSCCs than in normal tissues. Moreover, high expression levels of ITGB1, LAMA3, PLEC, and

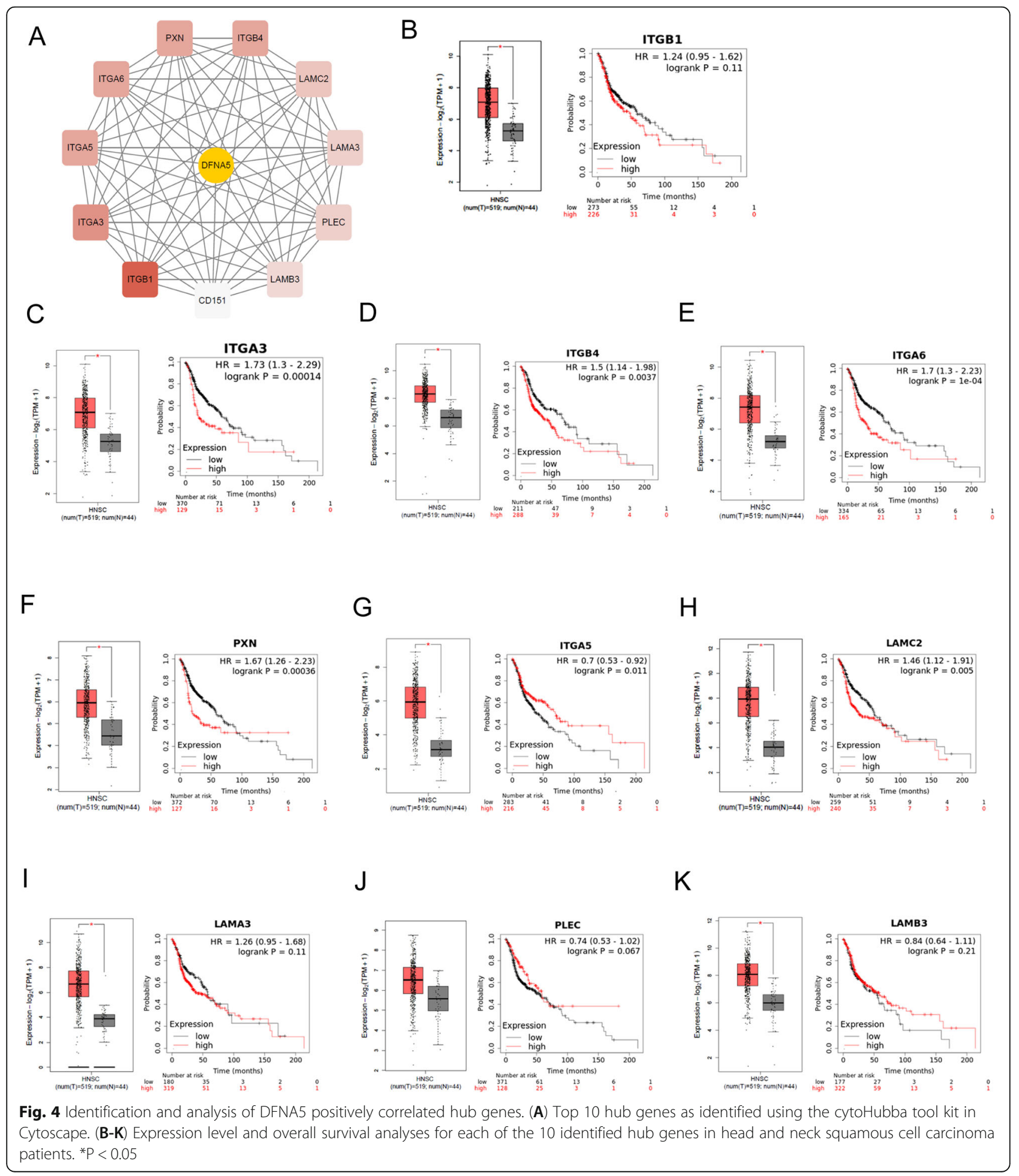




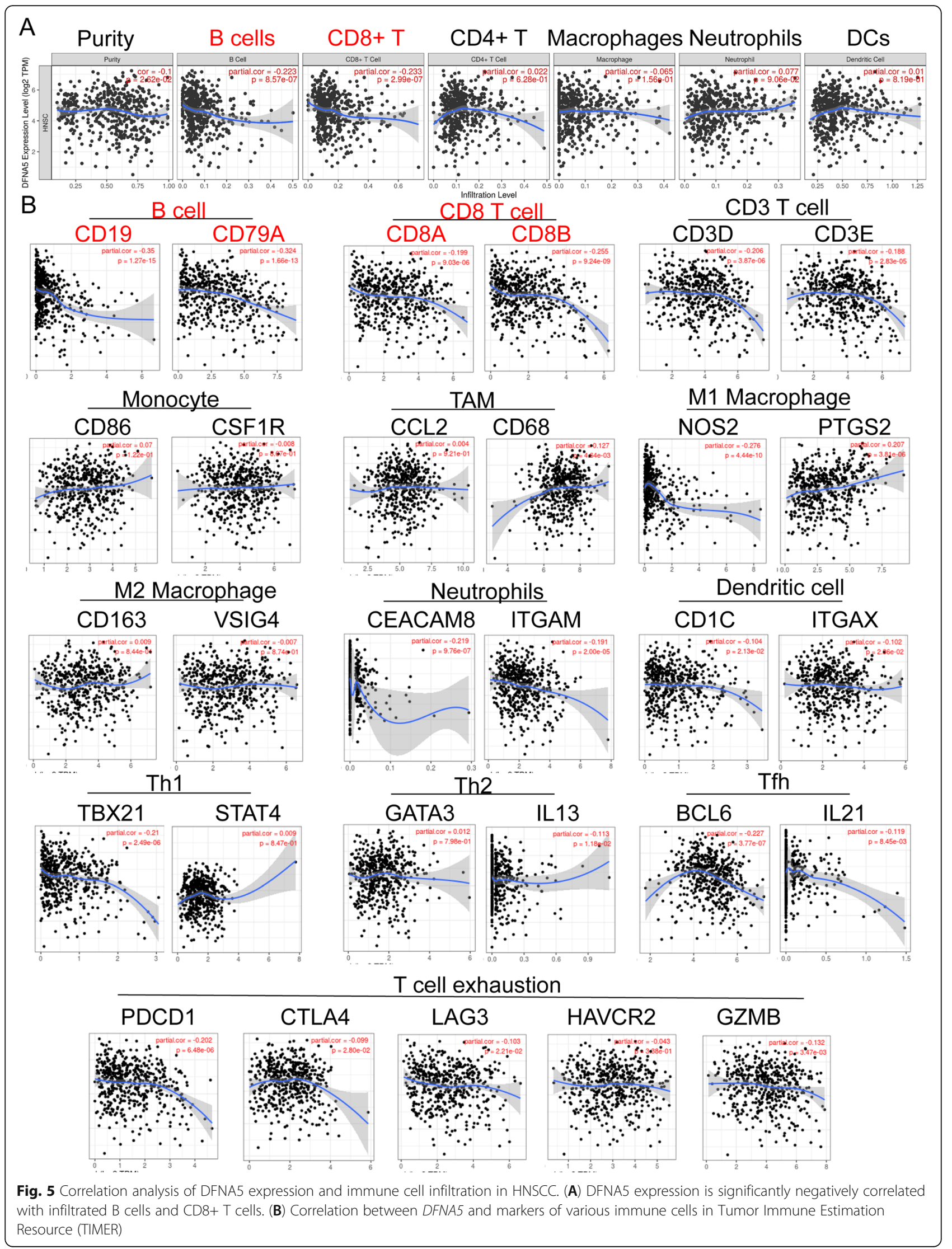


$L A M B 3$ correlated with poor OS in HNSCC patients (Fig. 4B-K). These results further support that DFNA5 mainly affects tumor progression in HNSCC by regulating cell adhesion through processes such as integrinrelated molecular pathways.

\section{High DFNA5 expression results in decreased lymphocyte infiltration in HNSCC}

To further explore the function of DFNA5 in HNSCC and its prognostic potential, we focused on the infiltration of immune cells in the HNSCC tumor microenvironment. Specifically, we used TIMER to investigate whether the expression of DFNA5 is related to the level of immune cell infiltration. Figure $5 \mathrm{~A}$ shows that the DFNA5 expression level was significantly negatively correlated with the infiltration of lymphocytes in HNSCC, such as $B$ cells (cor $=-0.223, P=8.57 \mathrm{e}-07$ ) and CD8 T cells (cor $=-0.223, P=2.99 \mathrm{e}-07$ ). Furthermore, we analyzed the correlation between DFNA5 expression levels and various immune cell markers in HNSCC, including subsets of T cells, B cells, M1 and M2 macrophages, neutrophils, natural killer cells, and dendritic cells. The results showed that the DFNA5 expression level was significantly negatively correlated with $\mathrm{B}$ cell and CD8 T cell markers (Fig. 5B). We conclude that a high level of DFNA5 expression significantly correlates with decreased tumor local lymphocyte infiltration, which is an important negative prognostic factor in HNSCC patients.

\section{Discussion}

HNSCCs are aggressive cancers originating from the epithelial mucosa of the digestive tract [20,21]. Although many studies have focused on the macro-genomic differences underlying the heterogeneity of these cancers, the survival rate of patients has not improved in the past decade [22]. Here, we showed that DFNA5 exerts oncogenic effects in HNSCC, as it is consistently overexpressed in cancer tissues and is an indicator of poor prognosis. Moreover, GO and KEGG pathway analyses showed that the upregulation of DFNA5 in HNSCC mainly affects cell adhesion by regulating processes such as integrin binding, and GSEA prediction shows the same results (Fig. 3G). These results are consistent with the fact that two cell-to-cell adhesion genes that act as tumor suppressors, namely CTNNA2 and CTNNA3, are frequently mutated in laryngeal carcinoma [23-25]. These adhesion proteins and their related pathways provide new candidate targets for formulating novel therapeutic strategies.

This study suggests that DFNA5 expression negatively correlated with lymphocyte infiltration in HNSCC patients. Changes in the immune system of HNSCC patients suggest that tumorigenesis is a comprehensive immunosuppressive process [26]. In the peripheral blood, the overall number of white blood cells in patients with HNSCC is decreased, and inhibitory $\mathrm{T}$ cells (Treg) become dominant among them [27]. Moreover, tumor-infiltrating lymphocytes (TILs) have been detected in many types of solid tumors, including HNSC Cs. In HNSCC patients, TILs have stronger anti-cancer activity than peripheral blood Treg cells $[28,29]$. Recent studies have shown that B cells and plasma B cells located in tumors or the tumor-draining lymph nodes play an important role in the formation of anti-tumor immune responses [30]. Moreover, $\mathrm{T}$ cells and B cells interact and coordinate their selection, specialization, and clonal expansion in tumor-associated tertiary lymphoid structures [31, 32]; the resulting plasma B cells are crucial to the anti-tumor immune response. As TILs are a major factor in this process, it comes as no surprise that their immunoprofile is a prognostic marker of disease-specific survival [33]. For example, in colorectal cancer, higher $\mathrm{CD} 8 / \mathrm{CD} 4$ ratios are associated with longer disease-free survival [34]. We found that DFNA5 expression was significantly negatively correlated with lymphocyte infiltration, especially of B cells and CD8+ T cells, suggesting that DFNA5 overexpression may exert its oncogenic function by hindering the anti-tumor immune response.

Notably, in contrast to our findings for HNSCC, DFNA5 expression is suppressed in many cancers, and reduced DFNA5 levels are associated with decreased survival in patients with breast cancer, suggesting that DFNA5 might be a tumor suppressor. In fact, recently study determined that the expression level DFNA5 in breast cancer patients was significantly decreased [35]; therefore, we speculate that DFNA5 plays a different role in different tumor types.

In conclusion, this is the first study to report DFNA5 as a new biomarker for HNSCC. More importantly, our results suggest an underlying mechanism for the oncogenic function of DFNA5, namely the reduction of immune cell infiltration through the modulation of cell adhesion. With further understanding of its functional scope, DFNA5 may become an effective tool for the diagnosis and treatment of HNSCC, and may help to make biomarker therapy a promising therapeutic option.

\section{Supplementary Information}

The online version contains supplementary material available at https://doi. org/10.1186/s12885-021-08692-w.

Additional file 1 Sup Fig. 1 DFNA5 transcription analysis in sub groups of patients with HNSCC.

Acknowledgements

Not Applicable. 


\section{Authors' contributions}

All authors participated in the drafting of the article or its critical revision for important intellectual content and approved the publication of the final version of the manuscript. $H T$, LZ and YL had full access to all the study data and take responsibility for data integrity and the accuracy of data analysis results. ZL, HL, QD, HL and BZ contributed to the conception, study design, and data collection.

\section{Funding}

This study was supported by The National Nature Science Foundation of China (NSFC81672659, NSFC81472523), Guangzhou Science and Technology Program Project Collaborative Innovation Major Projects (201704020112), Guangdong Natural Science Foundation (2020A1515010291), and Guangdong Basic and Applied Basic Research Foundation (2020A1515010315); Guangdong Medical Science and Technology Research Fund (A2020186) and Innovation Development Project of the First Affiliated Hospital, Guangzhou University of Chinese Medicine (2019QN01) to Yufeng Liu; Traditional Chines Mecdicine Bureau Of Guangdong Province (20211013) and Medical Scientific Research Foundation of Guangdong Province (A2021373) to Limei Zhong.

\section{Availability of data and materials}

The data used to support the findings of this study are available from the corresponding author upon reasonable request. Direct web links of datasets about; Oncomine: http://oncomine.org; UALCAN: http://ualcan.path.uab.edu; The Human Protein Atlas: https://www.proteinatlas.org; cBioPortal: http:// cbioportal.org; LinkedOmics: http://linkedomics.org; TIMER: https://cistrome. shinyapps.io/timer/; HCCDB: http://lifeome.net/database/hccdb/home.html.

\section{Declarations}

Ethics approval and consent to participate

Not Applicable.

\section{Consent for publication}

All authors have agreed to be published.

\section{Competing interests}

The authors declare that they have no conflicts of interest.

\section{Author details}

'Department of Oral and Maxillofacial Surgery, Hospital of Stomatology, Sun Yat-sen University, 56 Lingyuanxi Road, Guangzhou 510055, China.

${ }^{2}$ Guangdong Province Key Laboratory of Stomatology, Guangzhou, China.

${ }^{3}$ Department of Operative Dentistry and Endodontics, Hospital of Stomatology, Sun Yat-Sen University, 56 Lingyuanxi Road, Guangzhou 510055, China. ${ }^{4}$ Guangzhou First People's Hospital, Guangzhou 510000, China. ${ }^{5}$ Department of Laboratory Medicine, Guangdong Second Provincial General Hospital, No. 466 Xingang Middle Road, Haizhu District, Guangzhou 510317, China.

\section{Received: 8 March 2021 Accepted: 16 August 2021}

Published online: 25 August 2021

\section{References}

1. Siegel RL, Miller KD, Jemal A. Cancer statistics, 2015. CA Cancer J Clin. 2015; 65(1):5-29. https://doi.org/10.3322/caac.21254.

2. Patel J, Nguyen S, Ogretmen B, Gutkind J, Nathan C, Day T. mTOR inhibitor use in head and neck squamous cell carcinoma: a meta-analysis on survival, tumor response, and toxicity. Laryngoscope Investig Otolaryngol. 2020;5(2): 243-55. https://doi.org/10.1002/lio2.370.

3. Cronin KA, Lake AJ, Scott S, Sherman RL, Noone AM, Howlader N, et al. Annual report to the nation on the status of Cancer, part I: national cancer statistics. Cancer. 2018;124(13):2785-800. https://doi.org/10.1002/cncr.31551.

4. Cramer JD, Burtness B, Le QT, Ferris RL. The changing therapeutic landscape of head and neck cancer. Nat Rev Clin Oncol. 2019;16(11):669-83. https:// doi.org/10.1038/s41571-019-0227-z.

5. Van Laer L, Huizing EH, Verstreken M, van Zuijlen D, Wauters JG, Bossuyt PJ, et al. Nonsyndromic hearing impairment is associated with a mutation in DFNA5. Nat Genet. 1998;20(2):194-7. https://doi.org/10.1038/2503.
6. de Beeck K, Van Laer L, Van Camp G. DFNA5, a gene involved in hearing loss and cancer: a review. Ann Otol Rhinol Laryngol. 2012;121(3):197-207. https://doi.org/10.1177/000348941212100310.

7. Wu M, Wang Y, Yang D, Gong Y, Rao F, Liu R, et al. A PLK1 kinase inhibitor enhances the chemosensitivity of cisplatin by inducing pyroptosis in oesophageal squamous cell carcinoma. EBioMedicine. 2019;41:244-55. https://doi.org/10.1016/j.ebiom.2019.02.012.

8. Chen L, Weng B, Li H, Wang H, Li Q, Wei X, et al. A thiopyran derivative with low murine toxicity with therapeutic potential on lung cancer acting through a NF-kB mediated apoptosis-to-pyroptosis switch. Apoptosis. 2019; 24(1-2):74-82. https://doi.org/10.1007/s10495-018-1499-y.

9. Yu J, Li S, Qi J, Chen Z, Wu Y, Guo J, et al. Cleavage of GSDME by caspase-3 determines lobaplatin-induced pyroptosis in colon cancer cells. Cell Death Dis. 2019;10(3):1-20.

10. Toruner GA, Ulger C, Alkan M, Galante AT, Rinaggio J, Wilk R, et al. Association between gene expression profile and tumor invasion in oral squamous cell carcinoma. Cancer Genet Cytogenet. 2004;154(1):27-35. https://doi.org/10.1016/j.cancergencyto.2004.01.026.

11. Li T, Fan J, Wang B, Traugh N, Chen Q, Liu JS, et al. TIMER: a web server for comprehensive analysis of tumor-infiltrating immune cells. Cancer Res. 2017; 77(21):e108-10. https://doi.org/10.1158/0008-5472.CAN-17-0307.

12. Ru B, Wong C, Tong Y, Zhong J, Zhong S, Wu W, et al. TISIDB: an integrated repository portal for tumor-immune system interactions. Bioinformatics. 2019;35(20):4200-2

13. Gao J, Lindsay J, Watt S, Bahceci I, Lukasse P, Abeshouse A, et al. The cBioPortal for cancer genomics and its application in precision oncology. In: AACR; 2016.

14. Tang Z, Li C, Kang B, Gao G, Li C, Zhang Z. GEPIA: a web server for cancer and normal gene expression profiling and interactive analyses. Nucleic Acids Res. 2017;45(W1):W98-W102. https://doi.org/10.1093/nar/gkx247.

15. Hou G-X, Liu P, Yang J, Wen S. Mining expression and prognosis of topoisomerase isoforms in non-small-cell lung cancer by using Oncomine and Kaplan-Meier plotter. PLoS One. 2017;12(3):e0174515.

16. Chin C-H, Chen S-H, Wu H-H, Ho C-W, Ko M-T, Lin C-Y. CytoHubba: identifying hub objects and sub-networks from complex interactome. BMC Syst Biol. 2014:8(S4):S11. https://doi.org/10.1186/1752-0509-8-S4-S11.

17. Dennis G, Sherman BT, Hosack DA, Yang J, Gao W, Lane HC, et al. DAVID: database for annotation, visualization, and integrated discovery. Genome Biol. 2003;4(9):R60. https://doi.org/10.1186/gb-2003-4-9-r60.

18. Consortium GO. The gene ontology (GO) project in 2006. Nucleic Acids Res. 2006;34(suppl_1):D322-6.

19. Chandrashekar DS, Bashel B, Balasubramanya SAH, Creighton CJ, PonceRodriguez I, Chakravarthi BV, et al. UALCAN: a portal for facilitating tumor subgroup gene expression and survival analyses. Neoplasia. 2017;19(8):64958. https://doi.org/10.1016/j.neo.2017.05.002

20. Wenig BMJMP. Squamous cell carcinoma of the upper aerodigestive tract: precursors and problematic variants. Mod Pathol. 2002;15(3):229-54.

21. Rassouli FB, Matin MM, MJTB S. Cancer stem cells in human digestive tract malignancies. Tumour Biol. 2016;37(1):7-21.

22. Stransky N, Egloff AM, Tward AD, Kostic AD, Cibulskis $K$, Sivachenko A, et al. The mutational landscape of head and neck squamous cell carcinoma. Science. 2011;333(6046):1157-60. https://doi.org/10.1126/science.1208130.

23. Fanjul-Fernández M, Quesada V, Cabanillas R, Cadiñanos J, Fontanil T, Obaya A, et al. Cell-cell adhesion genes CTNNA2 and CTNNA3 are tumour suppressors frequently mutated in laryngeal carcinomas. Nat Commun. 2013;4(1):1-9.

24. Yanagi $H$, Wang $L$, Nishihara $H$, Kimura $T$, Tanino $M$, Yanagi $T$, et al. CRKL plays a pivotal role in tumorigenesis of head and neck squamous cell carcinoma through the regulation of cell adhesion. Biochem Biophys Res Commun. 2012;418(1):104-9. https://doi.org/10.1016/j.bbrc.2011.12.142.

25. Song J, Zhang P, Liu M, Xie M, Gao Z, Wang X, et al. Novel-miR-4885 promotes migration and invasion of esophageal Cancer cells through targeting CTNNA2. DNA Cell Biol. 2019;38(2):151-61. https://doi.org/10.1089/ dna.2018.4377.

26. Jie H, Gildener-Leapman N, Li J, Srivastava R, Gibson S, Whiteside T, et al. Intratumoral regulatory T cells upregulate immunosuppressive molecules in head and neck cancer patients. Br J Cancer. 2013;109(10):2629-35. https:// doi.org/10.1038/bjc.2013.645.

27. Weed DT, Vella JL, Reis IM, Adriana C, Gomez C, Sargi Z, et al. Tadalafil reduces myeloid-derived suppressor cells and regulatory $T$ cells and promotes tumor immunity in patients with head and neck squamous cell 
carcinoma. Clin Cancer Res. 2015;21(1):39-48. https://doi.org/10.1158/10780432.CCR-14-1711.

28. Duray A, Demoulin S, Hubert P, Delvenne P, Saussez S. Immune suppression in head and neck cancers: a review. Clin Dev Immunol. 2010;2011.

29. Solomon B, Young RJ, Rischin D. Head and neck squamous cell carcinoma: Genomics and emerging biomarkers for immunomodulatory cancer treatments. In: Seminars in cancer biology, vol. 2018: Elsevier; 2018. p. 228-40.

30. Yarchoan M, Ho WJ, Mohan A, Shah Y, Vithayathil T, Leatherman J, et al, Effects of B cell-activating factor on tumor immunity. JCl Insight. 2020;5(10): e136417.

31. Bruno T. New predictors for immunotherapy responses sharpen our view of the tumour microenvironment. Nature. 2020;577(7791):474-6. https://doi. org/10.1038/d41586-019-03943-0.

32. Sharonov G, Serebrovskaya E, Yuzhakova D, Britanova O, Chudakov D. B cells, plasma cells and antibody repertoires in the tumour microenvironment. Nat Rev Immunol. 2020;20(5):294-307. https://doi.org/1 0.1038/s41577-019-0257-x.

33. Lalami Y, Awada A. Innovative perspectives of immunotherapy in head and neck cancer. From relevant scientific rationale to effective clinical practice. Cancer Treat Rev. 2016;43:113-23. https://doi.org/10.1016/j.ctrv.2016.01.001.

34. Flaherty DC, Lavotshkin S, Jalas JR, Torisu-Itakura H, Kirchoff DD, Sim MS, et al. Prognostic utility of immunoprofiling in colon cancer: results from a prospective, multicenter nodal ultrastaging trial. J Am Coll Surg. 2016;223(1): 134-40. https://doi.org/10.1016/j.jamcollsurg.2016.03.003.

35. Zhang Z, Zhang Y, Xia S, Kong Q, Li S, Liu X, et al. Gasdermin E suppresses tumour growth by activating anti-tumour immunity. Nature. 2020;579(7799): 415-20. https://doi.org/10.1038/s41586-020-2071-9.

\section{Publisher's Note}

Springer Nature remains neutral with regard to jurisdictional claims in published maps and institutional affiliations.

Ready to submit your research? Choose BMC and benefit from:

- fast, convenient online submission

- thorough peer review by experienced researchers in your field

- rapid publication on acceptance

- support for research data, including large and complex data types

- gold Open Access which fosters wider collaboration and increased citations

- maximum visibility for your research: over $100 \mathrm{M}$ website views per year

At $\mathrm{BMC}$, research is always in progress.

Learn more biomedcentral.com/submissions 